\title{
Quelques réflexions sur les biais d'observation lors des essais sur les modèles réduits de forage
}

\author{
Some thoughts about biassed observations during the testing \\ of small scale models of wellbores
}

\author{
F.-J. SANTARELLI \\ Elf Aquitaine
}

Rev. Franç. Géotech. n 59, pp. $33-41$ (avril 1992)

\section{Résumé}

Cet article présente quelques réflexions sur les biais et la réalité des observations qui peuvent être faites lors d'essais sur des modèles réduits de forages. Après une introduction qui expose l'importance de tels tests dans un contexte industriel, il liste les principales géométries utilisées. L'étude systématique des machines et conditions d'essais fait apparaître les biais potentiels que ces systèmes d'essais peuvent engendrer dans leurs diverses configurations. Une série d'exemples illustratifs est ensuite présentée dans les domaines de l'effet d'échelle et des modes de rupture.

\footnotetext{
Abstract

This paper presents some thoughts about the bias and the reality of the observations which can be made during the testing of small scale wellbores. After an introduction which explains the importance of such tests in an industrial environment, it lists the different geometries used. A systemetic study of the testing machines and of the testing conditions highlights the potential areas where bias may occur during the observations. A series of illustrative examples are given in the fields of scale effects and failure modes.
} 


\section{INTRODUCTION}

La pratique pétrolière se caractérise peut être plus que tout autre domaine d'application de la mécanique des roches par une difficulté extrême à accéder directement à l'objet étudié qui est situé à grande profondeur. De ce fait, les seuls moyens d'observation de l'ingénieur sont les méthodes indirectes (diagraphies instantanées ou différées, géophysique, etc.). Le problème de la stabilité des parois, par exemple, qui nous occupera ici se produit dans des trous ayant un diamètre de l'ordre de 20 à $45 \mathrm{~cm}$. Ceci rend délicate la détermination des causes des mécanismes d'instabilité dont les paramètres sont souvent incertains.

Face à ce problème, il apparaît très important d'être capable de travailler sur des modèles physiques, le plus souvent à échelle réduite, pour pouvoir appré. hender cette réalité. Ceux-ci offrent l'intérêt de permettre l'isolation et le contrôle des paramètres et ainsi d'identifier les réponses du système soumis à une sollicitation parfaitement connue. On notera aussi que les résultats obtenus sur ces modèles sont les seuls éléments véritablement capables de permettre l'évaluation et la validation des modèles de calcul à vocation de dimensionnement.

Néanmoins, il convient de garder présent à l'esprit le fait que le modèle réduit est toujours différent de la réalité qu'il est censé représenter. En effet, entre la sollicitation théorique et la roche, se trouve la machine d'essais qui, de par sa nature, peut induire de nombreux artefacts qui, s'ils ne sont pas reconnus, peuvent conduire à une conception erronée de la réalité. Ces concepts erronés seront particulièrement dangereux, d'une part parce qu'ils proviennent d'un biais de l'observation et non pas de la modélisation, et d'autre part parce que les données limitées des cas de terrains permettront difficilement de détecter l'erreur.

Le but de cette présentation est donc de cerner les enjeux dans ce domaine des essais sur modèles réduits. Pour ce faire, la réalité de l'ingénieur sera d'abord présentée avec ses implications technicoéconomiques, et les divers paramètres du problème de la tenue des parois seront listés. Une revue des divers systèmes de test sera ensuite faite, et les applications, avantages et inconvénients de chacun présentés. Enfin l'application de ces concepts sera illustrée par des exemples concrets dans deux domaines particuliers : celui de l'effet d'échelle et celui des modes de rupture. Dans chaque cas, un exemple d'artefact et un exemple de réalité seront présentés. Notons ici que ce qui est alors considéré comme une réalité est un résultat qui n'a pas pu être relié à un artefact lié au système d'essais et qui semble donc correspondre à une réaction vraie de la roche. Néanmoins, il convient d'apprécier que de nouvelles investigations pourraient transformer cette réalité en artefact.

\section{LE PROBLÈME DE TENUE DES PAROIS EN PRATIQUE}

Dans la pratique, les instabilités de parois en forage ont un impact économique fort dans la mesure où ils représentent en moyenne un surcoût de 10 à
$20 \%$ pour la réalisation de l'ouvrage. Ce surcoût est critique dans le contexte du développement de champs marginaux et/ou difficiles comme ceux situés dans les domaines des hautes pressions et des hautes températures qui poussent la technologie jusque dans ses derniers retranchements. Le besoin de moyens pour juger de façon fiable de la faisabilité d'un puits devient alors crucial. Dans ce domaine peut-être plus que dans d'autre, une connaissance des mécanismes de déstabilisation des parois de l'ouvrage se révèle donc chaque jour plus importante comme le mentionnait le rapport d'une commission internationale s'occupant entre autres de ces problèmes (MAURY, 1987).

Les principaux paramètres pouvant entraîner une déstabilisation des parois d'un puits, dont l'action de certains a été clairement mise en évidence par l'analyse de cas de terrains, sont listés par GUENOT (1987) qui mentionne :

- les contraintes en place souvent anisotropes qui entraînent des comportements souvent très différents pour des puits de déviations et d'azimuts de déviation différents (MAURY et SAUZAY, 1987) ;

- la densité de la boue qui peut être soit trop faible et entraîner un écaillage de la paroi du trou pouvant aller jusqu'à son effondrement ou trop importante et entraîner une fracturation de la formation :

- la nature de la réponse de la roche qui peut être soit fragile, soit ductile, soit réactive chimiquement et qui présente donc toute une gamme de comportements possibles ;

- la température qui, par le biais de gradients entre le puits et la formation. pourra y générer un surcroît de chargement et contribuer à une déstabilisation (GUENOT et SANTARELLI, 1989) ;

- la composition chimique du fluide de forage (boue) qui sera plus ou moins agressive vis-à-vis de la réactivité et de l'altérabilité des formations traversées (évaporites, argilites, etc.) :

- les caractéristiques physiques du fluide de forage qui selon sa viscosité, sa teneur en solide, sa rhéologie pourra contribuer à la tenue ou à la rupture de la formation

- le régime de circulation du fluide de forage et les manceuvres du train de tiges qui peuvent jouer un rôle déstabilisateur en créant des cycles de surpression/dépression souvent très néfastes ;

- enfin l'outil de foration et la vitesse d'avancement.

A cette liste de paramètres qui gouvernent plutôt la tenue du puits en termes de ruptures matricielles doit aussi s'ajouter une autre liste de paramètres qui induisent des instabilités localisées (par exemple des mouvements sur discontinuités) qui ont été mises en évidence très récemment dans les puits pétroliers par les observations de MAURY et SAUZAY (1989), mais qui restent encore insuffisamment étudiées.

A cette série de paramètres, il convient d'opposer la très grande difficulté à observer et donc à diagnostiquer en temps opportun une instabilité qui pourrait se révéler fatale pour la suite des opérations comme le montre le cas vécu rapporté par GUENOT (1990). Une autre conséquence de la difficulté d'observation est le fait que les paramètres du problème ne sont 
souvent connus qu'avec une grande marge d'incertitude, même si diverses méthodes permettant une estimation indirecte des propriétés de la roche existent (SANTARELLI et al., 1991). Un autre point augmentant la difficulté de prévoir les instabilités de parois est le caractère relativement fruste des modèles de prédiction et ce malgré de nombreux développements récents (MC LEAN et ADDIS, 1990).

On comprend donc l'intérêt grandissant porté par l'industrie pétrolière aux essais sur modèles réduits de forage dans le but:

- d'observer les causes, les signes précurseurs et la manifestation de la rupture d'une paroi dans des conditions de chargement et de géométrie aussi représentatives que possible des conditions réelles;

- de tester la validité et le domaine d'application des modèles de prédiction ;

- de mettre à l'épreuve diverses solutions pour remédier à ces instabilités tels des additifs pour fluide de forage modifiant ses caractéristiques physiques et/ou chimiques, etc.

Les trois sections suivantes vont donc passer en revue les divers systèmes permettant de tester des modèles réduits de forage.

\section{LES TUBES ÉPAIS (OU «CYLINDRES CREUX *)}

Les tests sur tubes épais ont été pratiqués depuis le début du siècle soit sur des roches (ADAMS, 1912), soit même sur des monominéraux (BRIDGMAN, 1918). Il s'agit alors de tester des éprouvettes cylindriques de roches contenant une cavité cylindrique coaxiale (fig. 1). L'éprouvette peut ainsi être chargée simultanément par une pression extérieure, une pression intérieure et une force axiale (HOSKINS, 1969) voire également par un gradient de température entre les parois internes et externes du tube (JOHNSON et GANGI, 1980), etc.

En ce qui concerne la géométrie des tubes épais, et notamment le rapport du rayon extérieur de l'éprouvette sur le rayon de la cavité $\left(r_{0} / r_{i}\right)$, l'étude expérimentale de ROBERTSON (1955) indique qu'il existe un seuil dont la valeur est 3 environ. Lorsque $r_{0} / r_{1}$ est inférieur à 3 , le comportement du tube à la rupture est violent et s'apparente alors à celui d'une coque. Lorsque $r_{0} / r_{i}$ est supérieur à 3 , le comportement à la rupture fait alors apparaître une phase d'écaillage de la paroi de la cavité qui s'initie pour des charges inférieures à celles provoquant la ruine définitive de l'éprouvette (fig. 2). DAEMEN et FAlRHURST (1970) indiquent des valeurs pour la pression d'initialisation de l'écaillage en paroi qui varient entre 0,6 et 0,85 fois la pression d'effondrement du tube. Plus récemment, KOOJMAN et al. (1991) indiquent, grâce à des observations sous scanner, des valeurs de 0,88 fois la pression de ruine définitive de l'éprouvette. Il convient néanmoins de signaler que même si le rapport $r_{0} / r_{1}$ est supérieur à trois, l'analogie d'un tube épais avec une cavité souterraine n'est pas parfaite. En effet, pour certaines conditions rhéo-

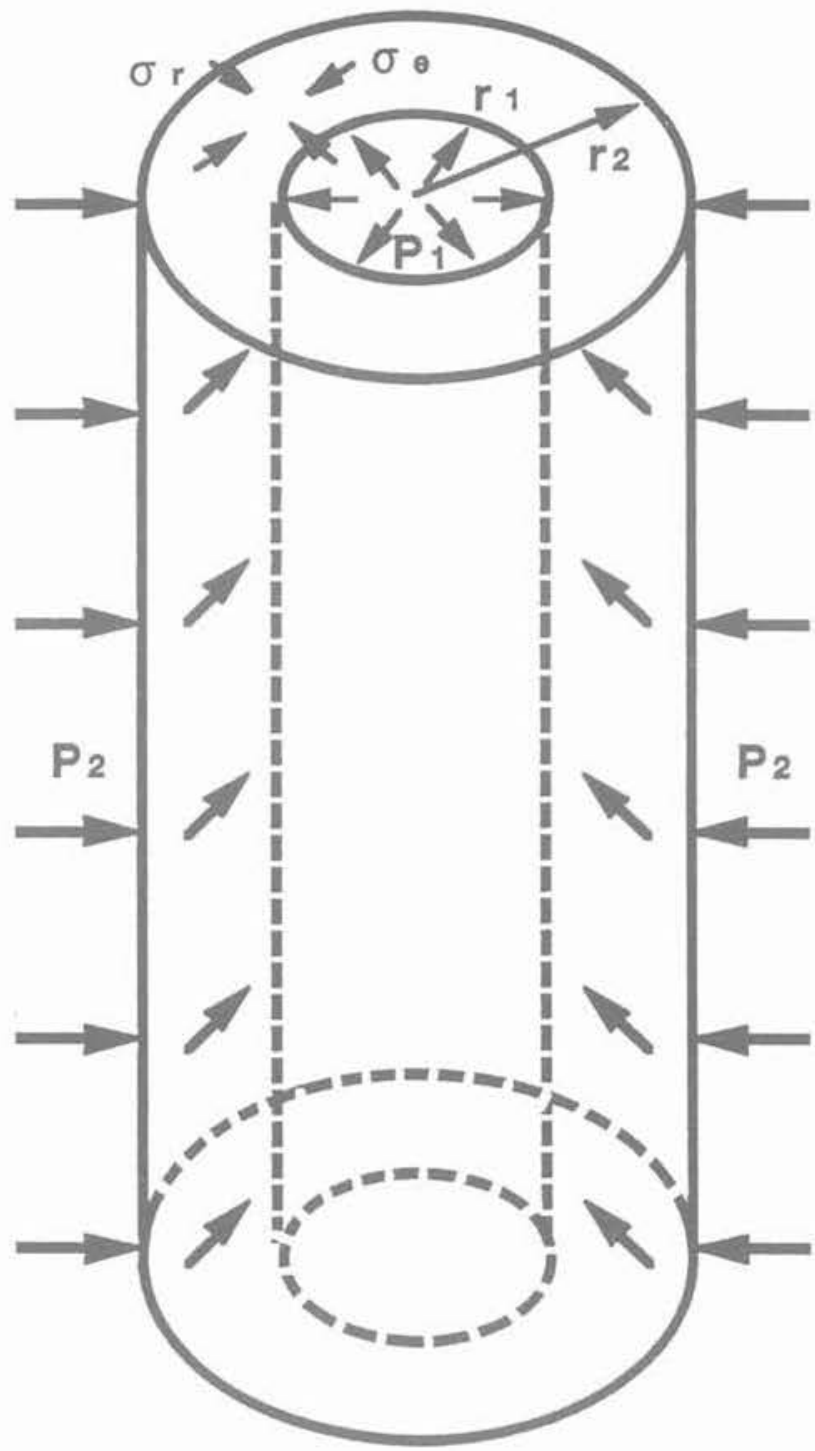

Fig. 1. - Schéma de principe des tubes épais.

Fig. 1. - Schematic of a thick walled hollow cylinder.

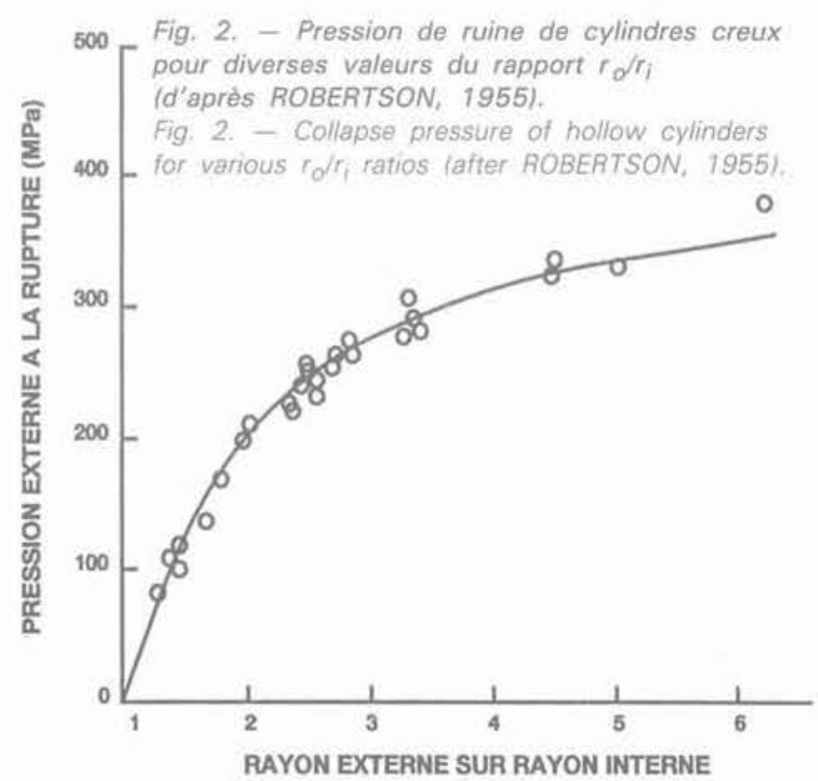


logiques du matériau, on peut calculer que le rayon d'influence d'une excavation souterraine peut être très important : par exemple 15 à 40 fois le rayon du trou pour un milieu particulaire, et jusqu'à 30 à 50 fois pour un milieu viscoplastique (DUSSEAULT, 1991). On conviendra aisément que de tels rapports $r_{0} / r_{1}$ sont difficilement utilisables au laboratoire et que le caractère fini de l'épaisseur du tube devra nécessairement être pris en considération pendant l'analyse et la modélisation des résultats des essais.

La longueur de l'éprouvette est habituellement choisie sur la base des méthodes recommandées par la SIMR (1981) pour les tests monoaxiaux et triaxiaux, c'est-à-dire un élancement de deux.

Parmi les avantages de cette géométrie, il convient de signaler que l'adaptation d'une cellule triaxiale classique pour effectuer des essais sur tubes épais est tout à fait minime à la seule condition que celle-ci ait été prévue pour recevoir des éprouvettes de taille suffisante (SANTARELLI, 1987), L'instrumentation de l'intérieur de la cavité qui est longtemps restée un problème a maintenant été réalisée de diverses manières que ce soit par jauges de déformations (SANTARELLI et BROWN, 1989), par mesure des variations de volume du fluide dans la cavité (COOK, 1970), ou enfin par l'utilisation d'extensomètres de type cantilever aujourd'hui disponibles commercialement. Finalement, l'essai sur tube épais présente l'avantage important d'être relativement peu affecté par le frettage losque le plateau par lequel la charge est appliquée suit la géométrie de l'éprouvette (BROWN et al., 1972).

Parmi les inconvénients de ce type d'essai, il convient de noter la difficulté d'observer la paroi de la cavité pendant le test et ainsi de déterminer avec précision le point d'initialisation de la rupture. Comme cette initialisation se produit souvent de façon très locale comme lillustre la photo de la figure 3 , elle n'a souvent aucun impact décelable sur la courbe de convergence moyenne de la cavité. Seule une mesure par jauge très locale ou un système d'image scanner permettent alors de la détecter. Un autre problème pratique concernant les tubes épais est celui de la concentricité de la cavité avec l'éprouvette qui ne peut jamais être parfaitement réalisée. Bien que GAY (1973) ait démontré que la perturbation liée à une faible excentricité soit très faible en termes de contraintes, il a été argumenté qu'elle pourrait néanmoins avoir beaucoup plus d'importance lorsque l'on traite du développement de la rupture. En effet, elle vient alors ajouter un défaut à la structure et pourrait ainsi conditionner le lieu où la rupture vient s'initialiser. Pourtant, les modes d'initialisation de l'écaillage autour de tubes épais pour plusieurs types de roches rapportés par SANTARELLI et BROWN (1989) montrent que la texture de la roche joue un grand rôle dans ce domaine, les conditions expérimentales même imparfaites ne jouant alors qu'un rôle plus ou moins secondaire.

\section{LES ESSAIS SUR BLOCS}

$\mathrm{Si}$ on se réfère à la liste des paramètres pouvant entraîner une instabilité en paroi du puits, on se rend immédiatement compte que la géométrie du tube épais est incapable de reproduire l'effet d'un état de contraintes en place anisotrope. Pour ce faire, il est nécessaire d'utiliser une machine triaxiale vraie et de tester des blocs dans lesquels on a, au préalable, percé une cavité cylindrique.

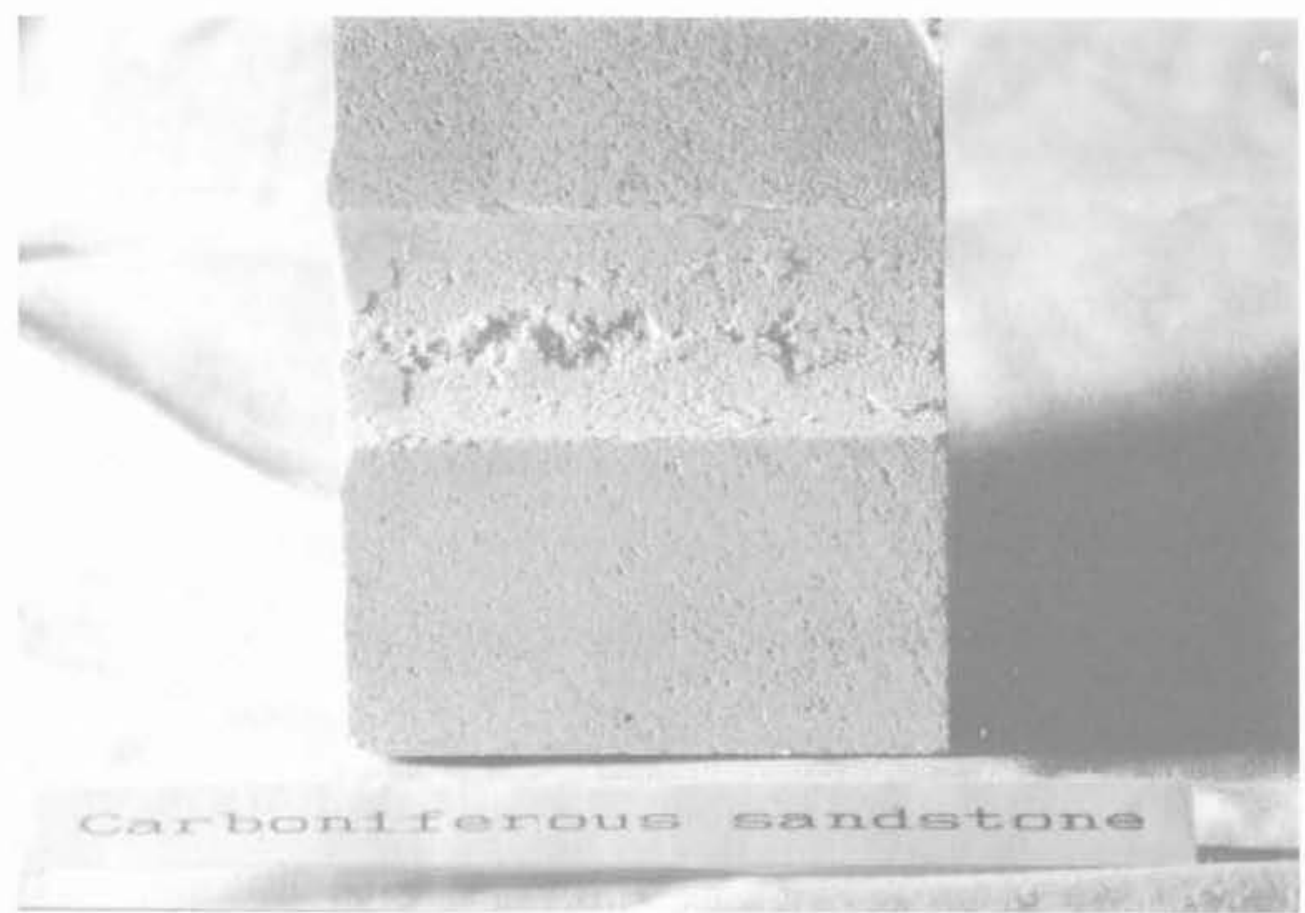

Fig. 3. - Initialisation de l'écaillage au mur d'un tube épais de grès carbonifère.

Fig. 3. - First signs of spalling at the wall of a thick walled hollow cyllinder of carboniferous sandstone. 
Diverses machines ont ainsi été développées. Il s'agissait au départ de simples cadres biaxiaux (KAISER et MORGENSTERN, 1981) puis de véritables machines triaxiales vraies basées sur le principe d'une application de la charge soit par piston (HENRY, 1988). soit par vérin plat (BANDIS et al., 1987). Suivant la technologie choisie, ces machines présentent des capacités très variables tant par la taille des blocs testés (13 cm d'arête pour la machine utilisée par HAIMSON et HERRICK (1985) jusqu'à $75 \mathrm{~cm}$ d'arête pour celle de HALLECK et al. (1988)) que par la charge maximum qu'elles peuvent appliquer. Notons que ce dernier point est tout à fait critique dans la mesure où les roches autour des cavités cylindriques vont se rompre pour des charges qui correspondent à des contraintes calculées par élasticité linéaire qui sont souvent très supérieures à la résistance à la compression uniaxiale de la roche (GUENOT, 1987).

Les problèmes liés à l'utilisation de telles machines sont assez nombreux et ont surtout été étudiés par ceux qui tentaient d'appliquer une charge biaxiale ou triaxiale à des prismes de roche. On notera tout d'abord celui du centrage de la charge qui est détaillé par MASO et LERAU (1980).

Le problème du frettage imposé par les pistons de charge à l'échantillon est lui aussi crucial. Par exemple, BROWN (1974) a démontré, en utilisant des plateaux à brosse, que l'effet apparent de la contrainte intermédiaire sur la résistance à la compression d'une : oche essayée en conditions biaxiales était très fortement dépendant de la technique utilisée pour appliquer la charge (fig. 4). Une étude a été menée par SOBOLEV et al. (1978), utilisant l'interférométrie holographique pour cartographier la déformation à la surface de plaques de pyrophyllite testées en condi-

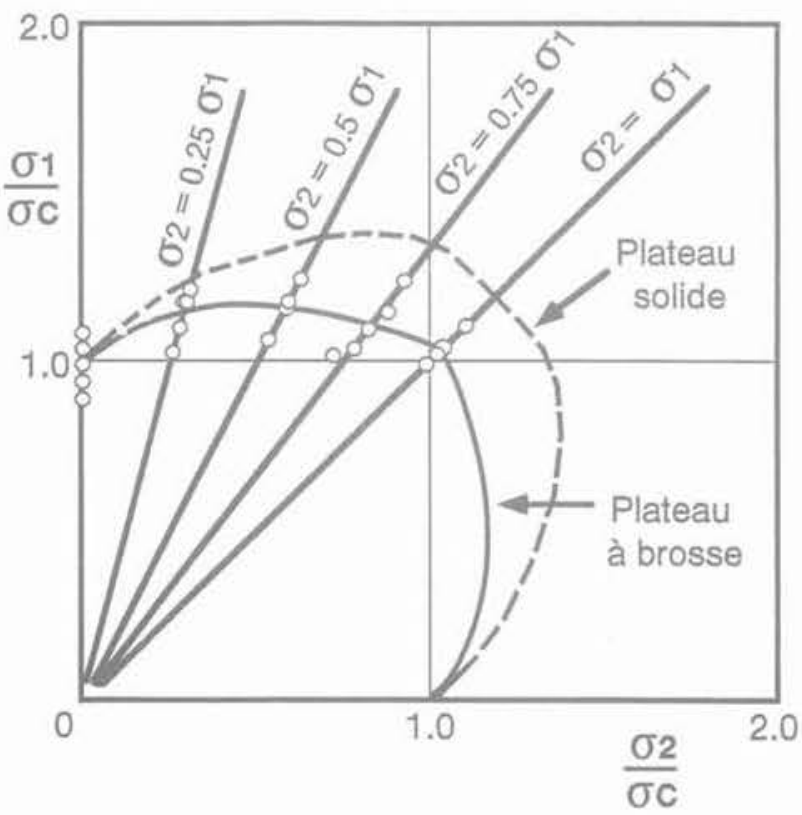

Fig. 4. - Variation apparente de la résistance du marbre de Wombeyan avec la contrainte intermédiaire pour deux types de frettage (d'après BROWN, 1974).

Fig. 4. - Apparent variation of the strength of Wombeyan marble. with the intermediate principal stress for two types of end effects (after BROWN, 1974). tions biaxiales; elle illustre parfaitement les conséquences d'un frettage mal maîtrisé. Ils montrent en effet que pour des contraintes appliquées variant entre 50 et $80 \%$ de la charge ultime, la déformation se concentre essentiellement le long d'une croix de SaintAndré reliant les coins de l'échantillon et est donc parfaitement hétérogène. ONAISI et al. (1990) ont montré l'influence du frettage, sur les modes de rupture obtenus dans des blocs percés d'un trou et testés en conditions monoaxiales, en faisant varier le rapport hauteur sur largeur de leurs éprouvettes.

A ces problèmes techniques vient s'ajouter le fait qu'il est très difficile de travailler en température et avec une pression de pore non nulle dans les blocs. D'autre part, il convient de reconnaître que ce type d'équipement est extrémement coûteux et donc encore assez peu répandu.

\section{LES BANCS DE FORAGE}

MAURY (1987) indique que l'état de la roche en paroi de galerie tel qu'il a pu être influencé par le creusement est un paramètre fondamental mais encore très mal apprécié pour la stabilité d'une excavation souterraine. Les deux méthodes d'essai envisagées ci-dessus peuvent difficilement tenir compte de cette action de forage et l'industrie pétrolière a été amenée à développer des moyens expérimentaux spécifiques pour étudier, entre autres, ce type de phénomène. Le principe du banc de forage sera donc présenté ici, car il vient en quelque sorte compléter la panoplie des moyens d'investigation.

Dans ce type de machines schématisé sur la figure 5 , une éprouvette de roche cylindrique de grande dimension est placée dans une cellule triaxiale et est soumise à une pression de confinement et à une pression de pore. Le piston utilisé pour appliquer la charge axiale est creux permettant ainsi le passage d'une tige et d'un outil de forage. Les conditions de forage d'une roche non seulement en termes géomécaniques (contraintes en places, pression de pore et température) mais aussi en termes de paramètres de forage (pression, température et débit de la boue, poids sur l'outil, vitesse de rotation, etc.) peuvent ainsi être reproduites.

Un tel banc de forage peut être utilisé soit directement pour simuler les conditions de forage soit avec des trous borgnes préforés dont on étudiera le comportement à long terme sous l'effet de diverses sollicitations. Par exemple, SALISBURY et al. (1991) utilisent un tel système pour étudier l'influence de la circulation d'un fluide à base d'eau dans un trou foré dans une argilite. Les principaux inconvénients de cette géométrie sont ceux qui ont été présentés dans la section consacrée aux tubes épais. Un problème particulièrement difficile dans le cas des bancs de forage est celui de l'instrumentation du trou. En effet, une boue de forage est un fluide abrasif, conducteur, corrosif en pression et en température, très hostile à tout instrument de mesure.

Après cette revue des moyens expérimentaux disponibles pour tester les modèles réduits de forage, por- 


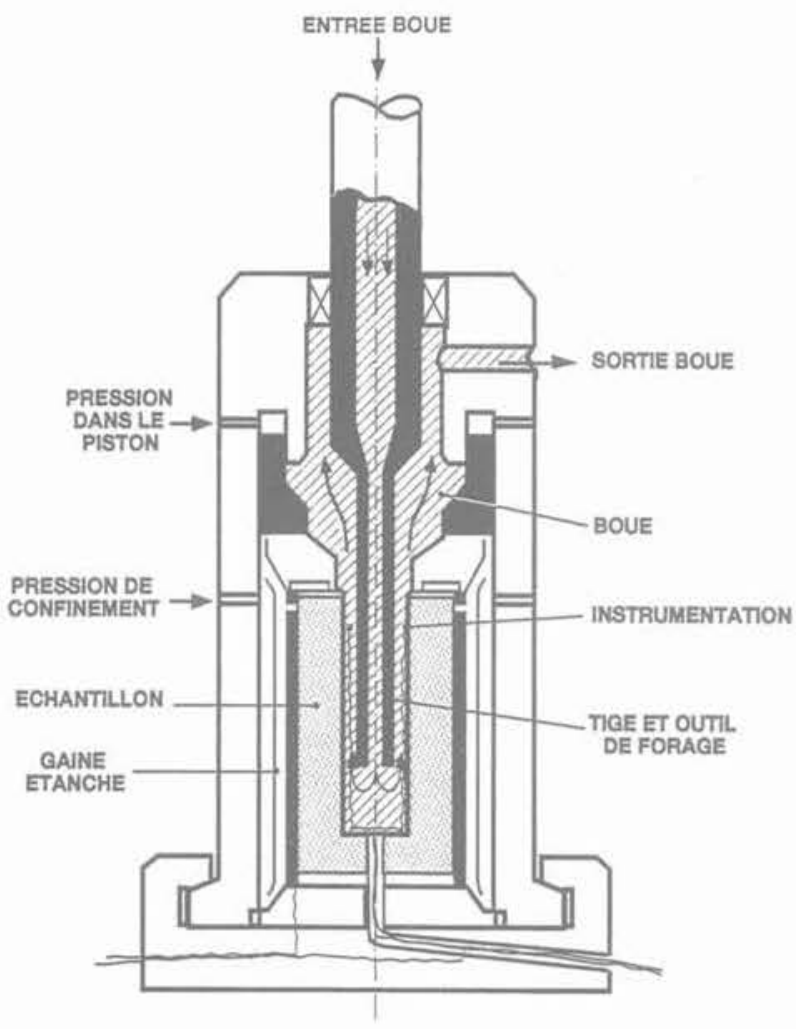

Fig. 5. - Schéma de principe d'un banc de forage. Fig. 5. - Schematic of a drilling bench.

tons notre attention sur la signification des résultats obtenus lors de telles études et notamment sur la part d'artefacts dus aux conditions expérimentales qu'ils peuvent contenir.

\section{EXEMPLE 1: L'EFFET D'ÉCHELLE}

Un premier exemple est celui de l'effet d'échelle. En mécanique des roches, il a été reconnu depuis longtemps que la taille de l'éprouvette testée pouvait grandement influencer la valeur des résultats d'essais telle celle de la résitance. En effet, on peut penser que plus le volume de roche testé est important et plus il y a de chances que celui-ci contienne des défauts qui vont venir l'affaiblir, et donc plus la valeur mesurée de la résistance du matériau sera faible (WEIBULL, 1939). Ce phénomène, souvent étudié et vérifié expérimentalement, (HABIB et VOUILLE, 1966 ; BERNAIX, 1969, etc.) a donc conduit de nombreux auteurs à suggérer que les trous de petit diamètre étaient plus stables que ceux de gros diamètre. Diverses séries d'essais visant à quantifier cet effet ont donc été menées.

HAIMSON et HERRICK (1989), ont utilisé des blocs cubiques de calcaire d'Alabama contenant une cavité cylindrique en leur centre. Dans tous les cas, le rapport entre l'arête du cube et le diamètre de la cavité était égal à 5 . Le chargement était appliqué suivant l'une des faces du cube par une machine d'essai classique. Ils ont ainsi observé que pour des diamètres de trou variant entre 2 et $12 \mathrm{~cm}$, la charge nécessaire pour provoquer l'apparition d'un écaillage en paroi variait de plus de 1 à 3 (fig. 6).

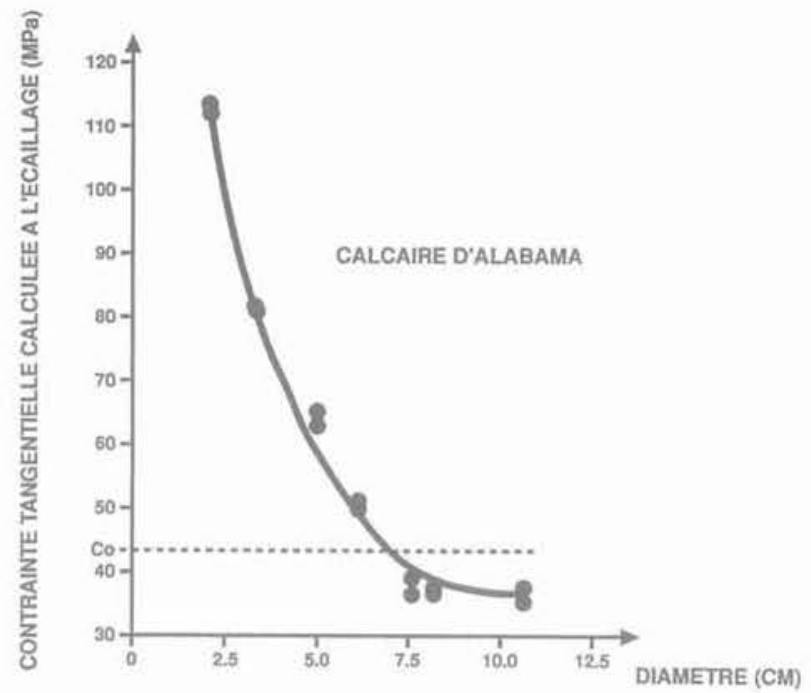

Fig. 6. - Effet d'échelle enregistré pendant des essais monoaxiaux sur blocs de calcaire d'Alabama (d'après HAIMSON et HERRICK, 1989). Fig. 6. - Scale effect measured during uniaxial tests of Alabama limestone blocs lafter HAIMSON and HERRICK, 1989).

RINGSTADT (1990) a réalisé une série d'essais sur des tubes épais de grès. Pour toutes les éprouvettes, le rapport entre le rayon de l'éprouvette et celui de la cavité était égal à 4 . Pour un rayon de cavité variant entre 1,25 et $5 \mathrm{~cm}$ lune des deux roches testées montrait un effet d'échelle faible (rapport de 1 à 1,3 ) alors que l'autre n'en montrait aucun (fig. 7).

Ces résultats apparemment contradictoires peuvent être clairement analysés lorsqu'on compare les conditions expérimentales utilisées par les deux auteurs suivant la méthode développée par BROWN et GONANO (1975). En effet, un effet d'échelle peut être grandement exagéré par l'utilisation d'un système expérimental imparfait notamment en termes de frettage. Or lappareillage utilisé par HAIMSON et

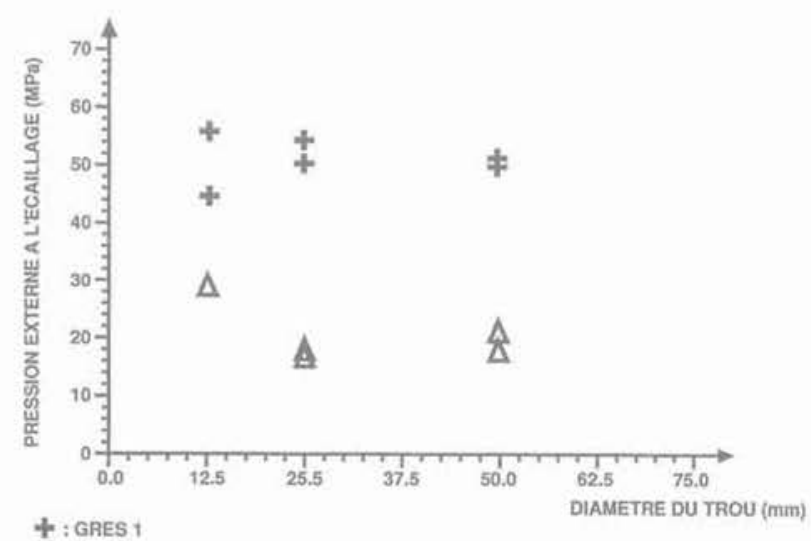

A: : GRES

Fig. 7. - Effet d'échelle enregistré pendant des essais sur tubes épais de deux grès. Mesures réalisées par IKU, Norvẻge (d'après RINGSTADT, 1990).

Fig. 7. - Scale effect measured during hollow cylinder tests on two sandstones. Measures performed at IKU, Norway lafter RINGSTADT, 19901. 
HERRICK (1989) est très proche de celui de l'essai monoaxial classique. L'utilisation d'une éprouvette cubique avec un rapport élancement largeur de 1 est donc tout à fait défavorable et il $y$ a de très fortes chances pour que la répartition des contraintes dans le bloc ait été très fortement perturbée par les conditions de frettage. Au contraire, les conditions de tube épais utilisées par RINGSTADT (1990) sont bien meilleures en terme de frettage. L'effet d'échelle très important rapporté par HAIMSON et HERRICK (1989) apparaît donc comme un artefact lié au matériel expérimental utilisé, alors que celui mesuré par RINGSTADT (1990) semble beaucoup plus proche d'un véritable comportement du matériau. D'une certaine façon, l'étude de KOOIJMAN et al. (1991) vient confirmer ce résultat et ce, bien que l'effet d'échelle qu'ils mesurent à partir d'essais sur tubes épais de grès de Castlegate soit plus important que celui rapporté par RINGSTADT (1990).

\section{EXEMPLE 2 : MODES DE RUPTURE}

Un autre exemple d'artefact est fourni par l'étude des modes de rupture susceptibles de se produire en paroi de puits et tels qu'on peut les observer lors d'essais sur tubes épais. Récemment, plusieurs auteurs (PERIE et GOODMAN, 1989 ; SANTARELLI et GUENOT. 1990) rapportaient la formation de fractures en extension suivant des plans $r-z$ dans les essais sur tubes épais. Pour tenter de vérifier la réalité d'un tel mode de rupture dans les conditions de charge utilisées par SANTARELLI et GUENOT (1990), une autre série d'essais a été conduite sur la même roche avec la même géométrie, pendant lesquelles, la décharge de l'éprouvette était très soigneusement contrôlée de façon telle que la contrainte axiale n'excédât jamais la pression appliquée par le fluide de confinement. Aucune des éprouvettes ainsi testées ne présente alors ces fractures en extension montrant ainsi qu'elles n'étaient, dans ce cas précis, que des artefacts. Néanmoins, les conditions d'essais de PERIE et GOODMAN (1989) qui travaillaient volontairement avec une contrainte axiale bien supérieure à la contrainte tangentielle peuvent avoir engendré de telles fractures tout comme celle d'ailleurs utilisées par HOSKINS (1969).

Un autre type original de rupture obtenu sur un tube épais est présenté sur la figure 8. La paroi présente alors un gauchissement local se traduisant par la formation d'une structure périodique sans que le moindre signe de macrofissure ne soit apparent dans cette région. Une telle observation qui n'avait encore jamais été rapportée pourrait aisément être interprétée comme un artefact ou " un hasard de la nature *. Néanmoins, l'éprouvette utilisée lors de l'essai où une telle rupture s'est produite avait été instrumentée par six jauges de déformation dans le but d'obtenir pour chaque niveau de chargement une carte aussi précise que possible de la convergence de la paroi du tube épais (fig. 9). Or, il se trouve que le gauchissement local du mur a eu lieu exactement sous l'une de ces jauges, et il est alors aisé de déterminer à quel moment de l'essai celui-ci s'est produit (fig. 10) et d'affirmer qu'il ne peut s'agir d'un artefact.

Les conditions de pression et de température régnant en parois au moment où le gauchissement s'est produit suggèrent qu'il pourrait s'agir de l'expression d'une déformation se produisant au moins dans le domaine de transition fragile/ductile, voire dans le domaine ductile.

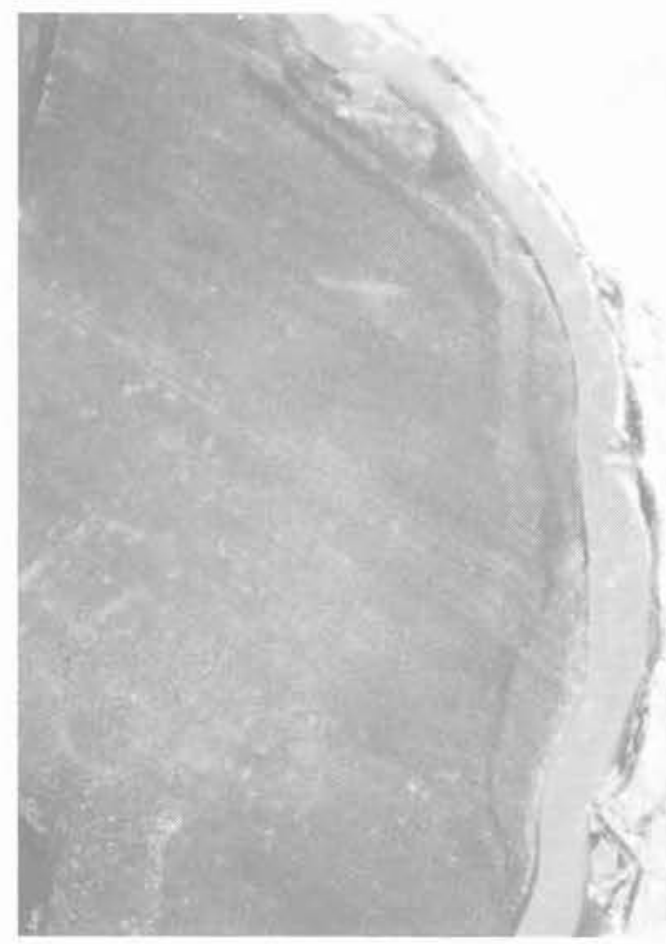

Fig. 8. - Gauchissement local du mur d'un tube épais.

Fig. 8. - Local buckling of the wall of a thick walled hollow cylinder. 


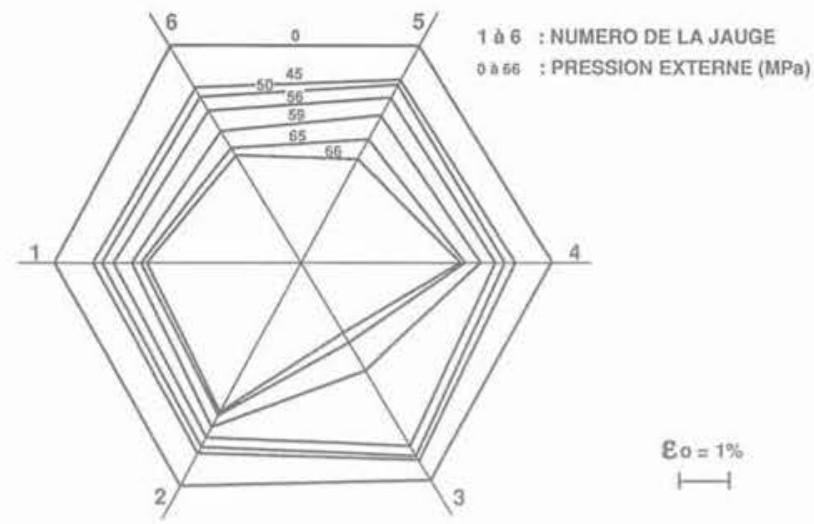

Fig. 9. - Profil de déformation du tube épais de la figure 8 sous divers niveaux de pression externe.

Fig. 9. - Convergence of the wall of the hollow cylinder from figure 8 for various outside pressures.

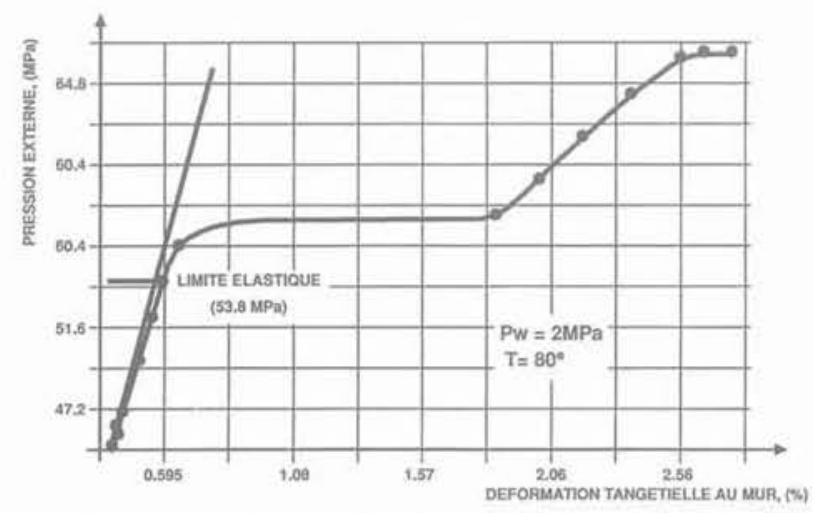

Fig. 10. - Déformation enregistrée par une jauge de déformation lors du gauchissement local du mur du tube épais de la figure 8 soumis à un accroissement monotone de la pression externe appliquée. Fig. 10. - Strain measured by a gauge during the local buckling of the wall of the hollow cylindre of figure 8 submitted to a continuous increase of its external pressure.

\section{CONCLUSION}

Après avoir brièvement mis en lumière l'intérêt des essais sur modèles réduits pour approfondir notre compréhension des problèmes de tenue des parois, un tour d'horizon des moyens expérimentaux a été fait. Il a été montré que, bien qu'ils soient complémentaires, les trois grands types d'essais (tubes épais, blocs et simulateurs de forage) présentaient tous divers problèmes expérimentaux qui, bien que déjà reconnus dans d'autres domaines plus classiques de la mécanique des roches, n'étaient pas complètement résolus (instrumentation, frettage, ...). En conséquence, l'analyse des résultats obtenus à partir de ces divers essais doit être faite avec un esprit critique certain dans le but de séparer ce qui pourrait procéder d'artefacts liés à un fonctionnement imparfait du dispositif expérimental et ce qui correspond effectivement à une réponse de la roche.

Plusieurs exemples illustratifs ont été présentés qui montrent que les principes à appliquer pour conduire et analyser les essais sur modèles réduits sont on ne peut plus classiques:
- s'assurer de l'homogénéité de la charge appliquée (que se passerait-il s'il n'y avait pas de cavité ?) :

- instrumenter les essais autant que faire se peut sans craindre une redondance des mesures dans le but de déterminer le seuil d'apparition d'hétérogénéité du champs de déformation qui accompagne très souvent la rupture.

Enfin, notons que la nature, de par sa complexité, réserve sans doute encore bien des surprises dans ce domaine relativement nouveau de la mécanique des roches.

\section{REMERCIEMENTS}

L'auteur voudrait remercier ELF AQUITAINE pour l'autorisation de publier cet article. Divers partenaires industriels qui ont été impliqués dans la réalisation et/ou le financement de certains résultats présentés ici doivent aussi être remerciés (AGIP, BP, IKU, SHELL, STATOIL). Enfin, le Prof. DUSSEAULT, les Dr MAURY et FOURMAINTRAUX ainsi que MM. DARDEAU et GUENOT qui ont contribué par divers commentaires, idées et résultats à cet article sont aussi remerciés.

\section{BIBLIOGRAPHIE}

ADAMS F.D. (1912), An experimental contribution to the question of depth of the zone of flow in the Earth's crust. J. Geol., 20, pp. 97-118.

BANDIS S.C., LINDMAN J., BARTON N. (1987), Three dimensional stress state and fracturing around cavities in overstressed weak rock. Proc. 6th ISRM Congr., (G. HERGET et S. VONGPAISAL, eds.), BLAKEMA, 2, pp. 769-775.

BERNAIX J. (1969), New laboratory methods of studying the mechanical properties of rocks. Int. J. Rock Mech. Min. Sci., 6 pp. 43-90.

BRIDGMAN P.W. (1918), The failure of cavities in crustals and rocks under pressure. Am. J. Sci., 45 , pp. $243-269$.

BROWN E.T. (1974), Fracture of rock under uniform biaxial compression. In Advances in Rock Mechanics, Proc. 3rd ISRM Congr., 2, Nat. Acad. Sci., Washington, pp. 111-117.

BROWN E.T., GONANO (1975), An analysis of size effect behaviour in brittle rock. Proc. 2nd Aust. New Zealand Conf. on Geomech., Inst. Engrs. Aust., Nat. Publ. No 75/4, pp. 139-143.

BROWN E.T., HUDSON J.A., HARDY M.P.. FAIRHURST C. (1972), Controlled failure of hollow rock cylinders in uniaxial compression. Rock Mech., 4, pp. 1-24.

COOK N.G.W. (1970), An experiment proving that dilatancy is a pervasive volumetric property of brittle rock loaded to failure. Rock Mech. Engng. Geol., 2, pp. 181-188.

DAEMEN J.J.K., FAIRHURST C., Influence of failed rock properties on tunnel stability. In Dynamic Rock Mechanics, Proc. 12 th U.S. Symp. Rock Mech., (G.B. CLARK, ed.), Soc. Min. Engrs. AIME, New York, pp. 855-875.

DUSSEAULT M.B. (1991), (Communication personnelle). 
GAY N.C. (1973). Fracture growth around openings in thick walled cylinders of rock subjected to hydrostatic compression. Int. J. Rock Mech. Min. Sci. \& Geomech. Abstr., 10, pp. 209-233.

GUENOT A. (1987), Contraintes et ruptures autour des forages pétroliers.Proc. 6th ISRM Congr., (G. HERGET et S. VONGPAISAL, eds.), BAL. KEMA, 1, pp. 109-118.

GUENOT A. (1990), General report : instability pro. blems at great depth drilling boreholes and wells. In Rock at Great Depth, Proc. ISRM/SPE Symp., (V. MAURY et D. FOURMAINTRAUX, eds.), BALKEMA, 3, pp. 1199-1208.

GUENOT A., SANTARELLI J.F. (1989), Influence of mud temperature on deep borehole behaviour. In Rock at Great Depth, Proc. ISRM/SPE Symp.. (V. MAURY et D. FOURMAINTRAUX, eds.). BALKEMA, 2, pp. 809-817.

HABIB P., VOUILLE G. (1966), Sur la disparition de l'effet d'échelle aux hautes pressions. C. R. Ac. Sci., 21 mars.

HAIMSON B.C., HERRICK C.G. (1985), In situ stress evaluation from borehole breakouts - Experimental studies. In Research and Engineering Applications in Rock Masses, Proc. 26 th US Symp, on Rock Mech., (E. ASHWORTH, ed.), BALKEMA, Rotterdam, pp. 1207-1218.

HAIMSON B.C., HERRICK C.G. (1989), Borehole breakouts and in situ stress. Proc. Drill. Symp. 12th An. Energy Sources Conf. ASME, (J.C ROWLEY, ed.), ASME, New York, pp. 17-22.

HALLECK P.M., SAUCIER R.J., BERHRMANN L.A., AHRENS T. (1988), Reduction of jet penetration in rock under stress. Paper SPE 18245, $15 \mathrm{pp}$.

HENRY J.P. (1988), (Communication personnelle),

HOSKINS E.R. (1969), The failure of thick walled hollow cylinders of isotropic rock. Int. J. Rock Mech. Min. Sci., 6, pp. 99-125.

JOHNSON B., GANGI A.F. (1980), Thermal cracking of non uniformaly heated thick walled hol. low cylinders of Westerly granite. Proc. 21st US Symp. Rock Mech., pp. 197-206.

KAISER P.K., MORGENSTERN N.R. (1981), Time dependent deformation of small tunnels : I. Expe. rimental facilities. Int. J. Rock Mech. Min. Sci. \& Geomech. Abstr., 18, pp. 129-140.

KOOIJMAN A.P. VAN DEN ELZEN M.G.A., VEEKEN C.A.M. (1991), Hollow cylinder collapse : measurement of deformation and failure in an Xray $C T$ scanner, observation of size effect. In Rock Mechanics as a Multidisciplinary Science, Proc. 32nd US Symp. on Rock Mech., (J.C. ROEGIERS, ed.), Balkema, Rotterdam, pp. $657-666$

MASO J.C.. LERAU J. (1980), Mechanical behaviour of Darney sandstone (Vosges, France) in biaxial compression. Int. J. Rock Mech. Min. Sci. \& Geomech. Abstr., 17, pp. 109-115.

MAURY V. (1987), Observation, recherches et résultats récents sur les mécanismes de rupture autour de galeries isolées. Proc. 6th ISRM Congr., (G.
HERGET et S. VONGPAISAL, eds.), BALKEMA, 2, pp. 1119-1128.

MAURY V., SAUZAY J.M. (1987), Borehole instability : case history, rock mechanics approach and results. Paper SPE/IADC 16051

MAURY V., SAUZAY J.M. (1989), Rupture de puits provoquée par glissement sur faille: cas vécu. mécanisme, remèdes, conséquences. In Rock at Great Depth. Proc. ISRM/SPE Sump.. (V. MAURY et D. FOURMAINTRAUX, eds.), BAL KEMA, 2, pp. 871-880.

MC LEAN M.R.. ADDIS M.A. (1990), Wellbore stability analusis : a review of current methods of analysis and their field application. Paper SPE/IADC 19941

ONAISI A., SARDA J.P.. BOUTECA M. (1990). Experimental and theoretical investigation of borehole breakouts. In Rock Mechanics Contributions and Challenges, Proc. 31st U.S. Symp. on Rock Mech., (W.A. HUSTRULID et G.A. JOHNSON, eds.), Balkema, Rotterdam, pp. 703-710.

PERIE P.J., GOODMAN R.E. (1989), Evidence of new failure patterns in a thick walled cylinder experiment. Proc. Drill. Sump. 12 th An. Energy Sources Conf. ASME, (J.C. ROWLEY, ed.), ASME, New York, pp. 23-27).

RINGSTADT C. (1990). (Communication personnelle).

ROBERTSON E.C. (1955), Experimental study of the strength of rocks. Bull. Geol. Soc. Am,, 66, pp. 1275-1314.

SALISBURY D.P., RAMOS G.G., WILTON B.S (1991), Wellbore instability of shales using a downhole simulation test cell. In Rock Mechanics as a Multidisciplinary Science, Proc. 32nd US Symp. on Rock Mech., (J.C. ROEGIERS, ed.), BALKEMA, Rotterdam, pp. 1015-1024.

SANTARELLI F.J. (1987). Theoretical and experimental investigation of the stability of the axisym. metric wellbore, $\mathrm{PhD}$ Thesis, University of London. $472 \mathrm{pp}$.

SANTARELLI F.J., BROWN E.T. (1989), Failure of three sedimentary rocks in triaxial and hollow culindrer compression tests. Int. J. Rock Mech. Min. Sci. \& Geomech. Abstr., 26 pp. 401-413.

SANTARELL.I F.J., DUSSEAULT M.B.. YASSIR N. (1991), Rock properties for petroleum engineers. Problem statement. Report of the ISRM/SPE Commission.

SANTARELLI F.J., GUENOT A. (1990), Experimental observations of bifurcation phenomena in rocks. In Rock at Great Depth. Proc. ISRM/SPE Symp., (V. MAURY et D. FOURMAINTRAUX, eds.), BALKEMA, 3, pp. 1563-1570).

SIMR (1981), Rock characterization testing and monitoring: ISRM Suggested Methods, (E.T. BROWN, ed.), Pergamon Press, Oxford, 211 pp.

SOBOLEV G., SPETZLER, SALOV B. (1978), Precursors to failure in rocks while undergoing anelastic deformations. J. Geophys. Res., 83, pp. 1775-1784.

WEIBULL W. (1939), A statistical theory of the strength of materials. Ing. Vetenskaps Akad. Handl., 151, pp. 5-44. 